\title{
Selective effects of division of attention on discrimination conditioning
}

\author{
M. C. CARRILLO \\ Rush-Presbyterian-St. Luke's Medical Center, Chicago, Illinois \\ J. D. E. GABRIELI \\ Stanford University, Stanford, California \\ and \\ J. F. DISTERHOFT \\ Northwestern University, Chicago, Illinois
}

\begin{abstract}
The contribution of attention to learning was examined in delay, trace, discrimination, and discrimination reversal eyeblink conditioning in young healthy adults. Participants performed either single- or two-cued conditioning paradigms with three levels of distraction: no distraction (full attention), concurrently watching a silent movie, or concurrent verbal shadowing. Conditioning for single-cue delay and trace conditioning paradigms was unaffected by the level of distraction. Conditioning for the two-cue paradigms of discrimination and discrimination reversal was reduced greatly when attention was divided. Silent movie watching allowed for some acquisition (CRs to the CS+) but no discrimination (differential responses to $\mathrm{CS}+$ vs. $\mathrm{CS}-$ ). Verbal shadowing eliminated both acquisition and discrimination. These results indicate that, in the human, attention makes a critical contribution to the successful acquisition of two-cue eyeblink conditioning tasks that require discrimination and discrimination reversal.
\end{abstract}

Classical eyeblink conditioning has been widely used to study associative learning in humans and animals (Gormezano, 1966). Different forms of eyeblink conditioning vary in their neural and psychological substrates. The delay conditioning paradigm involves the presentation of a tone conditioned stimulus (CS) that precedes and coterminates with a short airpuff unconditioned stimulus (US). Acquisition and retention of the CS-US association has been shown to depend on the cerebellum ipsilateral to the eye receiving the airpuff in rabbits (G. A. Clark, McCormick, Lavond, \& Thompson, 1984; McCormick \& Thompson, 1984a, 1984b; Yeo, Hardiman, \& Glickstein, 1985) and bilateral in humans (Daum et al., 1993; Solomon, Stowe, \& Pendlebury, 1989; Topka, Valls-Sole, Massaquoi, \& Hallett, 1992). Delay conditioning does not, however, depend on the hippocampus in rabbits (Akase, Alkon, \& Disterhoft, 1989; Schmaltz \& Theios, 1972; Solomon \& Moore, 1975) or on the temporal lobe in humans (Gabrieli et al., 1995).

Trace, unlike delay, conditioning demands mnemonic integration over time by introducing a silent interval between the tone (CS) and the airpuff (US). This temporal

This research was supported by Grant F31 GM 17223 to M.C.C. and by Grants NINDS IP50NS26985, NIH R01 AG08796, and NIH MH 47340 to J.F.D. Correspondence concerning this article should be addressed to M. C. Carrillo, Department of Neurological Sciences, Cognitive Neuroscience Section, Rush-Presbyterian-St. Luke's Medical Center, 1725 W. Harrison, Suite 1106 Pru. Bldg., Chicago, IL 60612 (e-mail: mcarrill@rush.edu). demand engages not only the brainstem and cerebellum, but also forebrain structures. Rabbits, rats, and humans with hippocampal lesions or medial temporal lobe damage are impaired in trace eyeblink conditioning (McGlincheyBerroth et al., 1997; Moyer, Deyo, \& Disterhoft, 1990; Solomon, Vander Schaaf, Thompson, \& Weisz, 1986; Weiss, Bouwmeester, Power, \& Disterhoft, 1999). These results suggest that the addition of the silent trace interval requires that the hippocampus be engaged in order to establish the CS-US association.

Delay and trace conditioning are examples of singlecue eyeblink conditioning - that is, a single tone (CS) followed by an airpuff (US). More complex forms of eyeblink conditioning, such as discrimination and discrimination reversal, involve two cues. Discrimination involves the presentation of two stimuli, one of which (the $\mathrm{CS}+$ ) is paired with a US, such as an airpuff; the other stimulus (CS-) is not paired with any US. This paradigm can be a delay or a trace discrimination procedure, depending on the duration and termination of the tone (CS) in relation to the airpuff (US). The present study used a delay discrimination and discrimination reversal paradigm in which relatively long tone CSs were used and the CS+ overlapped and coterminated with the airpuff US. During discrimination reversal, the significance of the tones was reversed, and the airpuff (US) was paired with the previously unreinforced CS. Acquisition of a two-tone discrimination remains intact in hippocampally lesioned rabbits (Berger \& Orr, 1983; Orr \& Berger, 1985). Hippocampectomized rabbits and humans with amnesia from medial 
temporal lobe lesions are able to acquire the new CS + during discrimination reversal but are unable to inhibit responses to the new CS - (Carrillo, Hopkins, et al., 1997; Orr \& Berger, 1985).

\section{AWARENESS IN EYEBLINK CONDITIONING}

A human participant can be consciously aware of something if and only if he or she can report it (Farber \& Churchland, 1995). The role of awareness in human eyeblink conditioning has been in contention for many years (LeBar \& Disterhoft, 1998). One empirical approach toward evaluating the role of awareness involved attempts to vary awareness of the CS-US contingency during conditioning (Cole, 1939; Grant, 1939). Such efforts, which involved manipulation of instruction sets with naive participants, were aimed primarily at determining the contribution of knowledge of stimulus contingencies to the conditioning process and at eliminating voluntary responses (conscious blinks to the tone CS given under voluntary control) to the CS. A voluntary response in human eyeblink conditioning is defined as a sharp, complete closure of the eye in response to the tone (CS), maintained until after termination of the airpuff (US; Spence \& Taylor, 1951). Several investigators devised methods by which to exclude voluntary responders from data sets (Gormezano \& Moore, 1962; Spence \& Ross, 1959), which along with neutral instructions, were employed to reduce the participant's ability to consciously control eyeblink responses. These studies showed that conditioning occurred in humans even when voluntary responders were eliminated and that participants who were not voluntary responders were still often aware of the CS- US contingencies. Disagreement on the role of awareness in conditioning has been prevalent in the literature. Some investigators have considered awareness of CS-US contingency essential to conditioning (Dawson \& Reardon, 1973; Kimble, 1971; Ross, 1971; Ross \& Nelson, 1973), and others have considered it unessential and even undesirable (Efron, 1966; Grant, 1973).

An alternative approach by which to determine the role of awareness in human conditioning was to diminish such awareness by use of a concurrent (or masking) task that divided participants' attention to the task (Ross, 1971). Attention has been referred to as selectivity in cognitive operations in which certain goals are in control of behavior and certain stimulus information is thereby relevant (Duncan, 1995). Discrimination learning was typically reduced when attention was divided by concurrent time estimation tasks or movie viewing (Mayer \& Ross, 1969; Ross \& Nelson, 1973). In contrast, concurrent task performance did not appear to reduce single-cue conditioning (unpublished study by Putnam reported in Ross, 1971). Ross concluded that discrimination learning may require an awareness of the CS-US elationship and that this awareness may be essential for some conditioning tasks and not others (Ross \& Nelson, 1973).

These findings raise the possibility that awareness of the relationship between the CS and the US may be essential for some more complex conditioning paradigms and that division of attention may selectively disrupt awareness and performance of those forms of conditioning. The foregoing studies treated attention as a unitary concept, but subsequent research has revealed that conditioning places demands on specific cognitive resources. Single-cue delay eyeblink conditioning is diminished by a concurrent tapping task but is not diminished by a concurrent verbal recognition task (Papka, Ivry, \& WoodruffPak, 1995). These results were interpreted as reflecting the essential role of cerebellar resources in eyeblink conditioning. Concurrent tapping competed for the same cerebellar resources as eyeblink conditioning - that is, circuitry involved in the timing of the response-whereas verbal recognition may not have competed for those resources. The idea that eyeblink conditioning and verbal learning involve separable memory systems was further supported by the finding that the magnitudes of delay conditioning and repetition priming were similar whether the two tasks were preformed concurrently or separately (Green, Ivry, \& Woodruff-Pak, 1999). The studies cited above show that specific cognitive resources, related to specific neural systems, are essential for conditioning.

More complex conditioning tasks, such as single-cue trace conditioning and two-cue discrimination and reversal, have not been extensively evaluated under divided attention conditions. R. E. Clark and Squire (1998) examined the role of awareness (conscious knowledge) of CS-US stimulus contingencies in delay and trace discrimination learning. The amnesic participants and their age-matched controls were older than those in most of the previous studies; they concurrently watched a silent movie in all conditions. Amnesic patients were intact for delay but impaired for trace discrimination (consistent with amnesic patients' being intact for delay but impaired for trace single-cue conditioning; Gabrieli et al., 1995; McGlincheyBerroth et al., 1997). For control participants, successful trace conditioning depended on awareness of the CS-US contingency (as elicited by a posttask questionnaire), but successful delay conditioning did not depend on such awareness. R. E. Clark and Squire (1998) concluded that trace discrimination eyeblink conditioning is a hippocampally dependent task of declarative memory and that conscious knowledge of stimulus contingencies is necessary for successful acquisition. Another study (R. E. Clark \& Squire, 1999) found that diminishing awareness with a concurrent visual digit detection task prevented acquisition of trace, but not of delay, discrimination conditioning. Informing participants of the stimulus contingencies prior to eyeblink conditioning resulted in successful performance of the delay, but not the trace, discrimination task, when the subjects simultaneously performed the digit 
Table 1

Age and Auditory Threshold

\begin{tabular}{|c|c|c|c|c|c|}
\hline \multirow[b]{2}{*}{ Group } & \multirow[b]{2}{*}{$n$} & \multicolumn{2}{|c|}{ Age } & \multicolumn{2}{|c|}{$\begin{array}{c}\text { Auditory } \\
\text { Threshold (dB) }\end{array}$} \\
\hline & & $M$ & $S D$ & $1 \mathrm{kHz}$ & $6 \mathrm{kHz}$ \\
\hline \multicolumn{6}{|c|}{ Discrimination-reversal } \\
\hline FA & 16 & 25.6 & 3.3 & 15.4 & 10.0 \\
\hline SM & 16 & 28.4 & 6.4 & 15.9 & 12.2 \\
\hline VS & 16 & 26.4 & 4.4 & 15.3 & 10.6 \\
\hline \multicolumn{6}{|l|}{ Delay } \\
\hline FA & 12 & 26.0 & 4.7 & 15.8 & \\
\hline SM & 12 & 27.5 & 5.4 & 18.8 & \\
\hline VS & 12 & 27.7 & 4.6 & 18.8 & \\
\hline \multicolumn{6}{|l|}{ Trace } \\
\hline FA & 12 & 26.7 & 3.7 & 16.3 & \\
\hline SM & 12 & 28.6 & 5.9 & 19.2 & \\
\hline vS & 12 & 27.3 & 5.3 & 16.3 & \\
\hline
\end{tabular}

Note-FA, full attention; SM, silent movie; VS, verbal shadowing.

detection task. In these discrimination studies, awareness of the CS-US contingency was essential for trace conditioning but irrelevant for delay conditioning.

The goal of the present study was to examine the role of attention for two dimensions of eyeblink conditioning that had previously been studied in isolation: temporal integration (the delay/trace contrast) and discrimination (the single-CS/double-CS contrast). The present study, therefore, examined the effect of dividing attention on four classical conditioning paradigms in young adult humans: two single-cue conditioning paradigms (delay and trace) and two double-cue paradigms (delay discrimination and delay discrimination reversal). In the full-attention condition, participants simply performed the conditioning task. In the divided-attention conditions, participants performed one of two concurrent tasks-viewing a silent movie or performing a verbal shadowing task. The silent movie procedure was originally described by WoodruffPak and Thompson (1988), who used the movie to alleviate boredom and improve alertness. Indeed, concurrent movie viewing has become a standard procedure in human eyeblink conditioning studies, as exemplified by the studies of R. E. Clark and Squire (1998, 1999). WoodruffPak and Thompson (1988) reported that conditioning performance was comparable between participants giving full attention and those concurrently watching a silent movie during single-cue delay conditioning. However, the effect of watching a silent movie during other types of eyeblink conditioning has not been systematically evaluated. Verbal shadowing is a more demanding concurrent task that requires participants to repeat, verbatim, a text being read aloud by an examiner.

The central question was whether division of attention would affect temporal integration (trace conditioning), discrimination, or both forms of conditioning. To our knowledge, this is the first direct examination of the consequence of dividing attention on single-cue delay versus trace conditioning and on single-cue versus dual-cue (discrimination) delay conditioning.

\section{METHOD}

\section{Participants}

The participants in this study were 120 normal adults, $20-40$ years of age, randomly assigned to one of three conditioning paradigms (see Table 1): delay-600, trace-500, or delay-600 discrimination and reversal. The participants in each of the paradigms were divided into one of three attention groups: full attention with no distraction, divided attention with a silent movie, and divided attention with a verbal shadowing task. The participants were recruited through advertisements posted at Northwestern University Medical School and Rush-Presbyterian-St. Luke's Medical Center. All the volunteers received a small payment for their participation.

\section{Apparatus}

The apparatus was a modified version of that previously used in our laboratory for rabbit eyeblink conditioning and extensively described elsewhere (Akase, Thompson, \& Disterhoft, 1994; Thompson, Moyer, Akase, \& Disterhoft, 1994). Eyeblink responses were measured with an infrared diode/photo transistor attached to an adjustable headband along with an airpuff delivery nozzle. The complete assembly was aimed at the right eye. Stimuli were delivered and responses analyzed by an IBM PC AT compatible microcomputer

\section{Procedure}

The volunteers were told they would be participating in an eyeblink experiment and were asked to sign an informed consent statement. They were seated and given a Folstein Mini-Mental Examination (MME; Folstein, Folstein, \& McHugh, 1975). Those participants who scored below the normal range of 28-30 were not included. None of the participants was excluded by this criterion. Each participant underwent an audiogram with a Beltone Model 119 audiometer. The participants in the delay and trace paradigms were tested at four frequencies: $0.5,1,2$, and $4 \mathrm{kHz}$. Participants in the discrimination and reversal paradigms were tested at five frequencies: $0.5,1,2,4$, and $6 \mathrm{kHz}$. If their hearing threshold was more than $15 \mathrm{~dB}$ above normal at 1 and $/$ or $6 \mathrm{kHz}$, they were not included in the study. Normal hearing threshold is $25 \mathrm{~dB} 0.0009$ newtons/ $\mathrm{cm}^{2}$ at these test frequencies (ANSI Standards, 1973). One participant was excluded by these criteria (see Table 1).

The participants were then fitted with the eyeblink apparatus and asked to make themselves comfortable. They were assigned to one of three attention conditions: full attention, in which the participant remained seated in a darkened room and was told to fixate on the television screen in front of her/him throughout the session; silent movie divided attention, in which the participant watched Charlie Chaplin's Gold Rush (Woodruff-Pak \& Thompson, 1988); and verbal shadowing divided attention, in which the participant listened to a story played aloud on a cassette player and was instructed to simultaneously repeat each word out loud. The story read was the introduction from The Tale of the Body Thief (Rice, 1992). The experimenter was seated in the same room, out of the participant's view, and read the following instructions:

Please listen carefully to the following instructions. Remain seated comfortably and fixate on the television screen in front of you, avoid ing eye movements such as looking around the room. Please try not to touch the headband or earphones at any time during the experiment. If you feel uncomfortable and feel that you want to adjust something, just let me know and I'll stop the experiment to make any adjustments you need. [Insert silent movie or verbal shadowing instructions here] You may communicate with me or quit the experiment at any time. Are these instructions clear to you? Do you have any questions?

[Silent movie instructions:] You will also be watching a silent movie during this session. Please relax and try to follow the story line, you will be asked a few questions about what went on in the movie afterwards. 
Table 2

Delay and Trace Mean Percent

Conditioned Responses, Amplitude (Amp), and Latency (Lat)

\begin{tabular}{|c|c|c|c|c|c|c|c|c|c|c|c|}
\hline \multirow[b]{2}{*}{ Group } & \multirow[b]{2}{*}{$n$} & \multicolumn{2}{|c|}{ Conditioning } & \multicolumn{2}{|c|}{ Extinction } & \multicolumn{2}{|c|}{ CR Amp (mV) } & \multicolumn{2}{|c|}{ UR Amp (mV) } & \multicolumn{2}{|c|}{ CR Lat (msec) } \\
\hline & & $M$ & $S D$ & $M$ & $S D$ & $M$ & $S D$ & $M$ & $S D$ & $M$ & $S D$ \\
\hline \multicolumn{12}{|l|}{ Delay } \\
\hline FA & 12 & 55.3 & 12.9 & 36.4 & 23.9 & $2,177.2$ & 589.4 & $2,839.3$ & 494.1 & 381.6 & 72.3 \\
\hline SM & 12 & 60.1 & 19.6 & 22.7 & 11.5 & $2,531.4$ & 455.7 & $2,933.9$ & 374.1 & 383.1 & 47.9 \\
\hline VS & 12 & 56.4 & 17.8 & 23.1 & 15.5 & $2,247.1$ & 606.5 & $2,788.9$ & 477.4 & 378.2 & 61.8 \\
\hline \multicolumn{12}{|l|}{ Trace } \\
\hline FA & 12 & 62.6 & 10.5 & 25.3 & 19.3 & $2,579.1$ & 624.9 & $3,120.2$ & 384.7 & 366.0 & 59.2 \\
\hline SM & 12 & 55.3 & 21.9 & 30.2 & 21.0 & $2,654.4$ & 823.6 & $2,718.6$ & 572.4 & 353.8 & 61.5 \\
\hline VS & 12 & 55.8 & 15.8 & 18.3 & 8.5 & $2,437.9$ & 464.9 & $2,579.2$ & 341.5 & 355.0 & 50.0 \\
\hline
\end{tabular}

Note - CR, conditioned response; UR, unconditioned response; FA, full attention; SM, silent movie; VS, verbal shadowing.

[Verbal shadowing instructions:] You will also be listening to a story during this session. Your task is to repeat each word you hear simultaneously out loud. Please try to follow the storyline, you will be asked a few questions about what happened in the story afterwards.

Parameters for delay conditioning were set as follows. The CS was an $85-\mathrm{dB}, 1-\mathrm{kHz}$ tone of $700-\mathrm{msec}$ duration and a $5-\mathrm{msec}$ rise/fall time, delivered binaurally with earphones, coterminating with a $100-$ msec 3 -psi corneal airpuff US to the right eye. Parameters for trace eyeblink conditioning were an $85-\mathrm{dB}, 1-\mathrm{kHz}$ tone of 100 -msec duration and a silent trace interval of $500 \mathrm{msec}$, which was followed by a $100-\mathrm{msec}$, 3-psi corneal airpuff to the right eye. Thus, the interval between onsets of the CS+ and the US was identical in the delay and trace paradigms. Discrimination and reversal parameters were the same as the delay- 600 described above with 1 and $5-\mathrm{kHz}$ tones as the $\mathrm{CS}+$ and $\mathrm{CS}-$. No airpuff was paired with the $\mathrm{CS}-\mathrm{CS}$ tone frequencies were counterbalanced among all the participants. The computer monitored eyeblinks for a baseline period of $750 \mathrm{msec}$ prior to the onset of the CS. In total, eyeblinks were monitored for $2,000 \mathrm{msec}$ per trial. The intertrial interval was $8-12 \mathrm{sec}$, with an average of $10 \mathrm{sec}$, which we have shown to support robust delay conditioning (Carrillo, Thompson, Gabrieli, \& Disterhoft, 1997).

Trace and delay conditioning sessions consisted of 5 puff-alone, 60 conditioning, and 30 extinction trials. Puff-alone trials were used to determine adequate unconditioned response (UR) amplitudes and correct position of the photocell diode. Conditioning trials consisted of paired CS and US presentations. Extinction trials consisted of tone-CS-alone presentations. After the session was completed, the participants were asked three questions about the silent movie or the verbal shadowing task. They were also asked to relate what the purpose of the experiment was in their own words.

Discrimination and reversal sessions consisted of 5 puff-alone trials and 120 discrimination and reversal trials. The first 60 trials were discrimination trials in which the participants received $30 \mathrm{CS}+$ and $30 \mathrm{CS}-$ randomized presentations. A CS+ trial was reinforced with a corneal airpuff; the CS - trial was not. Reversal consisted of 60 trials in which the significance of the tones was reversed. The 30 $\mathrm{CS}+($ previously $\mathrm{CS}-$ ) and $30 \mathrm{CS}-$ (previously $\mathrm{CS}+$ ) randomized presentations immediately followed the last trial of discrimination, with no time lapse except the intertrial interval. After the session was complete, the participants were asked three questions about the silent movie or the verbal shadowing tasks. They were also asked to relate what the purpose of the experiment was in their own words.

\section{Data Analysis}

An eyeblink four standard deviations greater than the mean baseline response amplitude, occurring after CS offset and before US onset, was defined as a conditioned response (CR). Spontaneous blink rate was monitored in the baseline period prior to the onset of the CS and was quantified to assess group differences. Blinks occurring during the first $100 \mathrm{msec}$ of the CS in the delay paradigm or during the $100-\mathrm{msec} \mathrm{CS}$ in the trace paradigm were considered alpha, or short-latency, responses and not CRs (Gormezano, 1966).

The mean numbers of CRs in the trace and delay paradigms were analyzed with a repeated measures analysis of variance (ANOVA; conditioning $\times$ distraction group) to assess group differences across blocks of five trials. Discrimination and reversal trials were analyzed by separating $\mathrm{CS}+$ reinforced trials and $\mathrm{CS}-$ unreinforced trials. For discrimination trials, repeated measures ANOVAs were performed to assess group differences on CS + and CS - trials separately across blocks of five trials. The mean number, latency, and amplitude of CRs, the mean amplitude of CRs, and the mean amplitude of URs were calculated for each subject for each paradigm and analyzed with a two-way ANOVA.

\section{RESULTS}

\section{Delay and Trace Conditioning}

All the participants in each group-no distraction, silent movie, and verbal shadowing - reported hearing the tone during the conditioning session. Performance was equivalent for delay and trace conditioning, and neither delay nor trace conditioning was affected by distraction. The mean percent CRs for the participants in each distraction group were analyzed with a repeated measures ANOVA across blocks of five trials (Table 2). A significant amount of overall conditioning occurred across blocks, as was evidenced by a significant effect for conditioning $[F(5,330)=11.484, p<.0001]$. There was no difference observed between delay and trace conditioning performance overall across blocks $[F(5,330)=0.87$, n.s. $]$, and no difference was observed between distraction conditions $[F(10,300)=0.84$, n.s.]. Mean percent CRs were also comparable for delay and trace paradigms, in all distraction conditions, as was evidenced by a lack of significance for the interaction of conditioning across blocks, paradigm and distraction task $[F(10,330)=0.61$, n.s.; Figure 1].

The quality of the CRs was also comparable in timing and size between the two paradigms, as well as among the three distraction groups. Table 2 shows the mean CR latency and amplitude for each group. No differences were observed for CR latency for delay or trace conditioning between distraction tasks, as was revealed by a one-way 

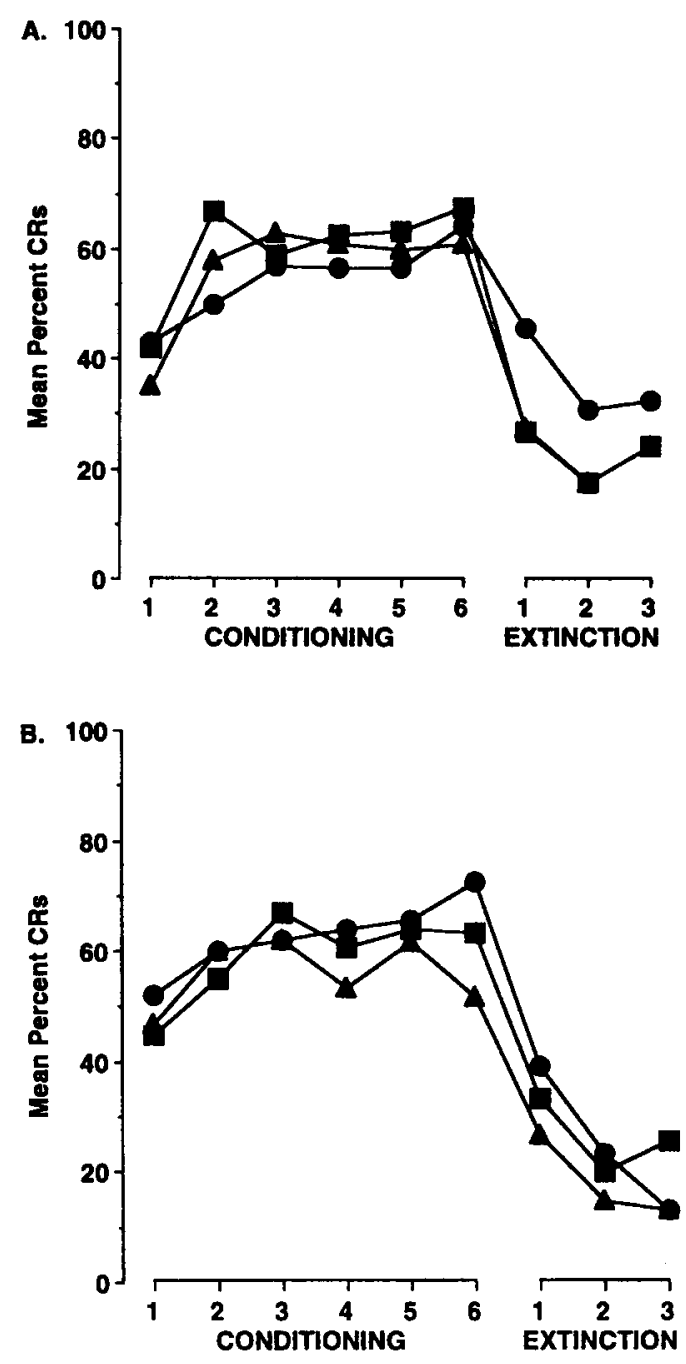

Figure 1. Delay (A) and trace (B) eyeblink conditioning in young controls under full-attention $(O)$ and silent movie $(\square)$ and verbal shadowing $(\boldsymbol{A})$ divided-attention conditions.

ANOVA. No significant effect was observed for delay and trace conditioning $[F(1,66)=2.63$, n.s. $]$, there was no effect for distraction task $[F(2,66)=0.10$, n.s. $]$, and there was no significant interaction $[F(2,66)=0.08$, n.s.]. No differences were observed for $\mathrm{CR}$ amplitude for delay or trace conditioning between distraction tasks: There were no significant differences for paradigm $[F(1,66)=2.78$, n.s.], for distraction group $[F(2,66)=1.20$, n.s.], or for the interaction $[F(2,66)=0.34$, n.s. $]$. No differences were observed for mean UR amplitudes for delay or trace between distraction groups, as was evidenced by a two-way ANOVA. No significant effect was found for paradigm $[F(1,66)=0.21$, n.s. $]$; there was no significant effect for distraction group $[F(2,66)=2.62$, n.s.], or for the interaction $[F(2,66)=2.43$, n.s. $]$.

\section{Delay and Trace Extinction}

All the groups comparably and successfully extinguished the acquired conditioned response [extinction, $F(2,132)=16.458, p<.0001 ;$ Figure 1$]$. The mean percent CRs for extinction in each distraction group is presented in Table 2. Mean percent CRs were analyzed with a two-way repeated measures ANOVA, with paradigm and distraction group as variables across blocks of five trials. No differences were found in rate of extinction for delay or trace paradigms between distraction groups, as was evidenced by no difference in extinction between paradigms $[F(2,132)=1.79$, n.s. $]$ or between distraction groups $[F(4,132)=2.31$, n.s.], and no differences were observed for the interaction $[F(4,132)=0.37$, n.s. $]$.

\section{Discrimination}

All the participants in each group-no distraction, silent movie, and verbal shadowing-reported hearing two distinct tones during the discrimination condition ing session. Significant differences were found in mean percent conditioned responses to the $\mathrm{CS}+$ during discrimination between distraction groups, mainly owing to a low performance by the verbal shadowing group (Figure 2). The mean percent CRs for discrimination and reversal across each distraction group are presented in Table 3. A two-way repeated measures ANOVA across blocks of five trials showed a significant main effect for group $[F(2,45)=15.83, p<.0001]$ and a significant interaction for group across blocks $[F(10,225)=2.81$, $p<.05]$. A Fisher's post hoc analysis revealed that the difference was due to lower performance by the verbal shadowing group than by the other two groups-full attention $(p<.0001)$ and silent movie $(p<.001$; Figure 2). A low CR level to the CS - was detected across all the groups, indicated by no group difference across blocks for responses $[F(2,45)=2.47, p>.05]$. The full-attention and silent movie groups adequately suppressed responses to the CS-, as was indicated by no difference being observed for CS - blocks $[F(10,225)=$ $0.57, p>.05]$.

Differences in amount of discrimination between the $\mathrm{CS}+$ and the $\mathrm{CS}-$ during the initial discrimination were assessed by subtracting mean percentage of CRs for the $\mathrm{CS}-$ from mean percentage for the CS+ (see Table 3 ). A stepwise reduction in amount of discrimination was observed, with the full-attention group exhibiting the most discrimination between $\mathrm{CS}+$ and $\mathrm{CS}-$, the silent movie group an intermediate amount, and the verbal shadowing group exhibiting the least amount of discrimination (Figure 2). A significant main effect was observed in the amount of discrimination between groups, as was evidenced by a one-way factorial ANOVA for group $[F(2,45)=9.99, p<.001]$. A Fisher's post hoc analysis showed that the amount of discrimination was significantly greater in the full-attention group than in the silent movie $(p<.01)$ and verbal shadowing $(p<$ 

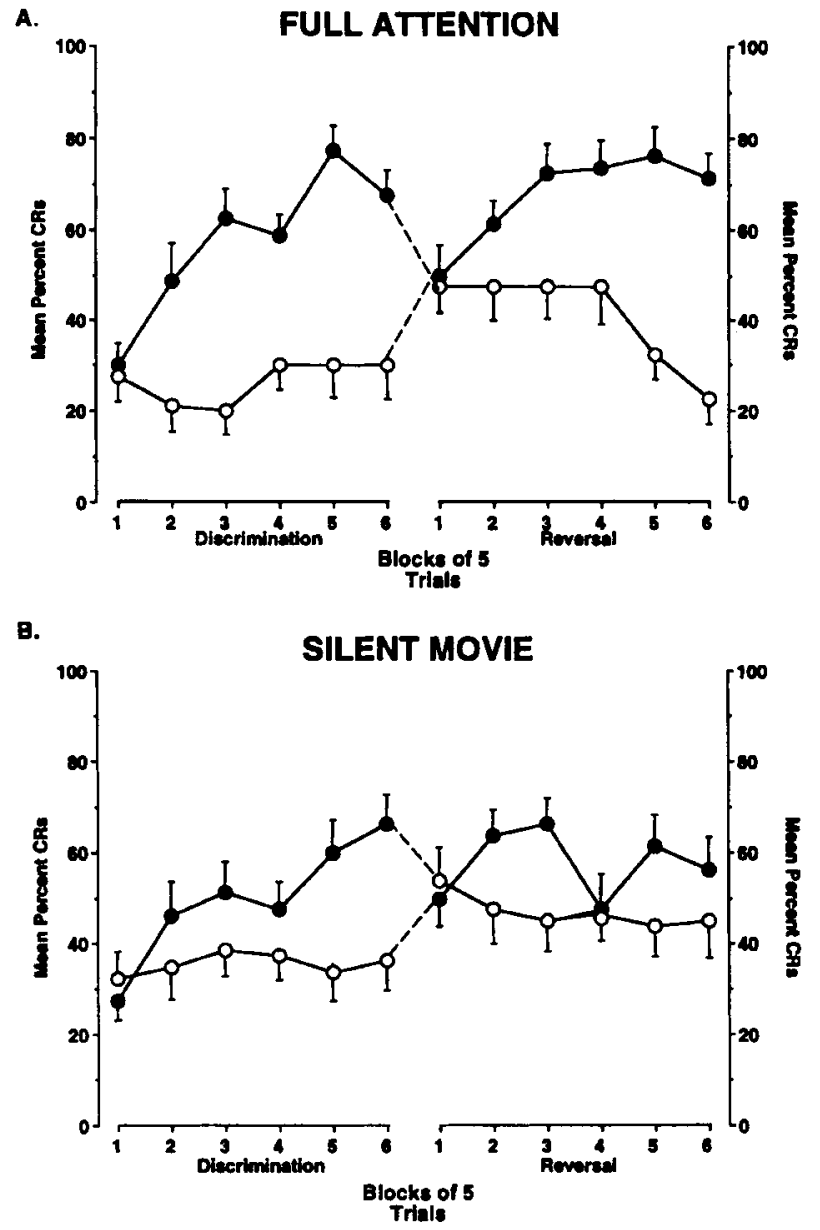

c.

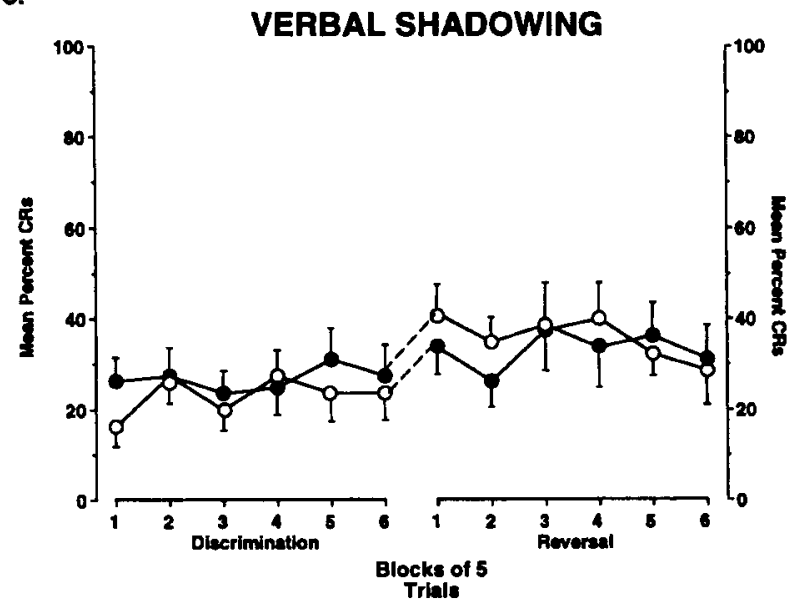

Figure 2. Discrimination and discrimination reversal in young controls under three attention conditions: full attention, silent movie, and verbal shadowing ( $O, \mathrm{CS}+$; $\mathrm{O}, \mathrm{CS}-$ ).

$.0001)$ groups and that there was no difference between the silent movie and the verbal shadowing groups.

Shorter CR latency and smaller CR amplitude, but no difference in UR amplitude, were observed during discrimination trials. Table 3 shows the mean CR latency and amplitudes for each group. A one-way ANOVA for $\mathrm{CS}+$ mean $\mathrm{CR}$ latency and $\mathrm{CR}$ amplitude during discrimination revealed a significant difference between distraction groups for latency $[F(2,45)=7.47, p<.05]$ and amplitude $[F(2,45)=4.95, p<.05]$. Fisher's post hoc tests indicate the differences were due to shorter latency and smaller amplitude responses in the verbal shadowing distraction group. No significant difference was found for UR amplitude $[F(2,45)=0.49, p<.05]$, indicating that the lower response rate and differences in size and timing of the CRs in the verbal shadowing group were not due to nonassociative factors or differences in placement of the photocell diode.

\section{Discrimination Reversal}

All the participants in each group-no distraction, silent movie, and verbal shadowing - reported hearing two distinct tones during the discrimination reversal conditioning session. Performance across blocks for the $\mathrm{CS}+$ during discrimination reversal revealed a significant difference in mean percent $\mathrm{CRs}$ to the $\mathrm{CS}+$ between the three distraction groups, as was evidenced by a significant group difference $[F(2,45)=10.02, p<.001$; Figure 2] and a significant interaction of distraction group and block $[F(10,225)=2.22, p<.05]$. A Fisher's post hoc test revealed that the difference was caused by a significant difference in mean percent CRs to the new $\mathrm{CS}+$ in the verbal shadowing group, as compared with the full-attention group $(p<.0001)$ and the silent movie group $(p<.001)$. There were no differences observed for mean percent $\mathrm{CRs}$ to the $\mathrm{CS}-$ between groups, as was evidenced by a lack of main effect for group $[F(2,45)=$ $1.25, p>.05]$, and no difference in the interaction, indicating reversal of the response in the full-attention and silent movie groups. (Note: Successful reversal was not seen for the verbal shadowing group, since no initial discrimination was observed; see Figure 2.)

Differences in amount of discrimination after the reversal were assessed by subtracting mean percentage of CRs for the CS - from mean percentage for the CS+during the reversal paradigm (see Table 3). The amount of discrimination during reversal conditioning was significantly different between distraction groups, as was evidenced by a significant group effect $[F(2,45)=11.05$, $p<.001]$. Again, a stepwise reduction in amount of discrimination during reversal was observed, as indicated by a Fisher's post hoc test in which the full-attention condition exhibited the greatest amount of discrimination between the CS+ and the CS - during reversal, as compared with the silent movie group $(p<.05)$ and the verbal shadowing group ( $p<.0001)$, and between the silent movie and verbal shadowing distraction groups $(p<.05)$.

Shorter CR latency and smaller CR amplitude, but no difference in UR amplitude, was observed during reversal trials. Table 3 shows the mean CR latency and amplitudes for each group. A one-way ANOVA for CS+ mean $\mathrm{CR}$ latency and amplitudes during discrimination reversal revealed a significant difference between distraction groups for CR latency $[F(2,45)=6.16, p<.05]$ and $C R$ 
Table 3

Discrimination and Reversal Mean Percent Conditioned Responses, Amplitude (Amp), and Latency (Lat)

\begin{tabular}{|c|c|c|c|c|c|c|c|c|c|c|}
\hline \multirow[b]{2}{*}{ Group } & \multicolumn{2}{|c|}{$\mathrm{CS}+$} & \multicolumn{2}{|c|}{$\mathrm{CS}-$} & \multicolumn{2}{|c|}{$\mathrm{CS}+\mathrm{CR}$ Amp (mV) } & \multicolumn{2}{|c|}{$\mathrm{CS}+$ UR Amp $(\mathrm{mV})$} & \multicolumn{2}{|c|}{ CS+ CR Lat (msec) } \\
\hline & $M$ & $S D$ & $M$ & $S D$ & $M$ & $S D$ & $M$ & $S D$ & $M$ & $S D$ \\
\hline \multicolumn{11}{|c|}{ Discrimination } \\
\hline FA & 57.5 & 13.0 & 29.6 & 15.5 & $2,158.7$ & 536.1 & $3,223.9$ & 628.0 & 387.6 & 40.2 \\
\hline SM & 49.8 & 18.1 & 35.6 & 15.7 & $2,209.5$ & 669.3 & $3,032.2$ & 558.8 & 387.7 & 55.8 \\
\hline VS & 26.8 & 16.5 & 23.8 & 14.1 & $1,594.4$ & 628.7 & $3,096.5$ & 477.5 & 309.7 & 87.9 \\
\hline \multicolumn{11}{|l|}{ Reversal } \\
\hline FA & 63.6 & 19.8 & 40.8 & 16.1 & $2,227.2$ & 721.0 & $2,844.3$ & 764.3 & 389.0 & 63.8 \\
\hline SM & 57.5 & 18.8 & 47.1 & 17.9 & $2,544.2$ & 713.3 & $3,012.3$ & 514.0 & 404.5 & 61.6 \\
\hline VS & 33.1 & 22.6 & 36.0 & 24.5 & $1,739.5$ & 681.3 & $2,949.4$ & 672.4 & 317.3 & 92.1 \\
\hline
\end{tabular}

Note-FA, full attention; SM, silent movie; VS, verbal shadowing.

amplitude $[F(2,45)=5.09, p<.05]$. A Fisher's post hoc test indicated that the differences were due to the short latency and small amplitude of responses in the verbal shadowing distraction group (Table 3 ). No significant difference was observed for UR amplitude $[F(2,45)=0.27$, $p>.05]$.

\section{Spontaneous Blink Rate}

Spontaneous blinks during the trace and delay paradigms were sampled during the 750 -msec baseline period of each trial. A two-way ANOVA revealed no difference in blink rate for paradigm $[F(1,66)=0.75, p>.05]$, a significant effect for distraction group $[F(2,66)=4.95$, $p<.05]$, and no interaction $[F(2,66)=0.56, p>.05]$. A Fisher's post hoc analysis indicated that the distraction group difference occurred between the silent movie and the verbal shadowing groups and that the verbal shadowing group had a significantly greater number of spontaneous blinks. The verbal shadowing group did not differ from the full-attention group in number of spontaneous blinks. More important, the spontaneous blinks did not interfere with acquisition in the verbal shadowing distraction group during delay and trace conditioning.

Spontaneous blinks for discrimination and reversal paradigms were quantified and analyzed with a two-way ANOVA. No significant differences were observed during discrimination trials $[F(2,45)=3.11$, n.s.] or for reversal $[F(2,45)=1.89$, n.s. $]$.

\section{Alpha Responses}

Alpha, or short latency, responses were quantified and analyzed for discrimination trials and reversal trials separately. A significant group difference was revealed for alpha responses in discrimination trials $[F(2,45)=4.0$, $p<.05]$. A Fisher's post hoc test indicated that the difference was due to an increased number of alpha responses in the verbal shadowing distraction group. No significant difference was observed for alpha responses during reversal $[F(2,45)=2.41$, n.s. $]$.

\section{Awareness}

The participants were given postsession interviews in which they were asked to relate what they thought the purpose of the experiment was. It is important to note that no method for assessing awareness after classical conditioning is optimal (Lebar \& Disterhoft, 1998). The participants were rated as aware of the stimulus contingencies in the trace and delay paradigms if they reported an association between the tone and the airpuff and cessation of the airpuff during extinction. The participants were rated as aware during discrimination and reversal if they reported an association between one of the tones and the airpuff, as well as reversal of the significance of the tones.

Seven of 72 participants in the delay and trace paradigms reported no tone-puff (CS-US) relationship and thus were labeled unaware: 2 in the delay-full-attention group, 1 in the delay-movie-distraction group, 1 in the delay-verbal-shadowing-distraction group, 0 in the trace-full-attention group, 2 in the trace-silent-moviedistraction group, and 1 in the trace-verbal-shadowingdistraction groups. A chi-square test revealed no significant difference in the proportion of aware versus unaware participants $\left[\chi(5)^{2}=2.69, p=.75\right]$.

Fourteen of 48 participants ( $n=16$ per group) reported that they were unaware of the $\mathrm{CS}+/ \mathrm{CS}-$ contingency in the discrimination and reversal paradigm: 2 in the fullattention group, 2 in the silent movie distraction group, and 10 in the verbal shadowing distraction group. A chisquare test revealed a significant group difference in the proportion of aware versus unaware participants $\left[\chi(2)^{2}=\right.$ $12.91, p<.001]$. A post hoc cell contribution table revealed that the verbal shadowing distraction group aware and unaware cells are those contributing to the significant differences in number of occurrences (verbal shadowing group $=3.59$ ). Awareness in the verbal shadowing group did not affect performance on discrimination and discrimination reversal, as was revealed by a repeated measures ANOVA performed on the 10 aware, as compared with the 6 unaware, participants $[F(1,14)=.14$, $p>.05]$.

\section{DISCUSSION}

In the present study, trace and delay single-cue conditioning was unaffected by dividing attention with either a silent movie or a verbal shadowing task. No difference was observed in level or rate of acquisition between groups. Since there are multiple dissociations between 
single-cue delay and trace eyeblink conditioning in animals and humans, we hypothesized that division of attention would impair trace, relative to delay conditioning. However, dividing attention by viewing a silent movie or by verbally shadowing an examiner did not diminish either delay or trace single-cue conditioning.

In contrast, acquisition of a two-tone discrimination and its reversal were impaired in both divided-attention conditions. Although the full-attention and silent movie groups showed significant acquisition to the CS + across blocks, both divided-attention conditions (silent movie and verbal shadowing) eliminated any significant difference in the amount of discrimination (difference in percentage of $\mathrm{CRs}$ between the $\mathrm{CS}+$ and the $\mathrm{CS}-$ ) during the initial acquisition and its subsequent reversal. Poor performance by participants in the verbal shadowing group during discrimination and reversal was primarily due to a lack of conditioned responding to either CS. However, when CRs did occur, they were of short latency and small amplitude. These nonadaptive CRs are similar to responses observed in hippocampectomized rabbits during 500msec trace conditioning (Moyer et al., 1990; Solomon et al., 1986). The robust effects of both divisions of attention on discrimination demonstrates that the lack of any such effects in the single-cue paradigms cannot be attributed to any weakness of the divisions of attention.

Attentional requirements differed greatly between the two divided-attention tasks in the present study. The silent movie task required only that the participant view the movie and remember a few events for subsequent testing. Consistent with Woodruff-Pak and Thompson (1988), we found that viewing the movie did not affect delay conditioning. The effect of silent movie viewing has not been previously evaluated for trace conditioning, discrimination, or discrimination reversal. Silent movie viewing selectively diminished conditioning on discrimination and discrimination reversal but had no effect on trace conditioning.

The verbal shadowing task is a much more attentiondemanding task than silent movie watching. Accordingly, concurrent performance of the shadowing task had a greater affect on discrimination learning; in fact, it prevented such learning altogether. Shadowing differs in at least two important ways from silent movie watching. First, there is competition for auditory processes between the speech of the examiner and the participant and the tone CS or CSs. Second, there could be competition for motor processes between the participant's speech and the eyeblink. Despite these potential competitions for auditory and motor resources, verbal shadowing had no effect at all on either delay or trace conditioning. This suggests that the particular motor demands of speech did not compete for cerebellar resources (otherwise, we would have found a reduction in delay conditioning, as Papka et al., 1995, did for a tapping task selected deliberately to invoke cerebellar timing processes). Verbal shadowing, however, eliminated discrimination learning altogether. This effect is unlikely to reflect competition for motor re- sources, because such a competition would have affected single-cue delay or trace conditioning. It may be that the need to discriminate between two tones requires finer auditory processing than do the single-cue conditions and that the auditory competition between tone perception and shadowing contributed to the elimination of discrimination conditioning. However, participants were asked in the postsession interview to describe the discrimination task, and all were able to report hearing two different tones during the conditioning session. Perhaps more important, neither auditory nor motor competition can account for the reduction of discrimination caused by the silent movie viewing.

Thus, the present results suggest that almost any substantial division of attention reduces delay discrimination learning. These findings are consistent with prior studies of delay discrimination, using other distraction tasks (Mayer \& Ross, 1969; Ross, 1971; Ross \& Nelson, 1973). Additional support for these findings comes from a study by Preston, Disterhoft, and Gabrieli (2000, personal communication), who found a disruption in delay two-tone discrimination, using a visual two-back task. Furthermore, the particular divisions of attention in the present study (silent movie viewing and verbal shadowing) produced a dissociation between two different forms of single-tone conditioning (delay and trace) and dual-tone discrimination. This suggests that discrimination per se requires a substantial degree of some as yet unspecified form of attention and that such attention is easily diminished by performance of a wide range of concurrent tasks. In terms of neural systems, the attentional resources critical for discrimination are unlikely to be associated with either cerebellar areas critical for delay conditioning or hippocampal areas critical for trace conditioning, because division of attention affected neither delay nor trace conditioning.

One goal of dividing attention is to diminish awareness of the CS-US contingency. Measuring such awareness is challenging because awareness could be defined in a variety of ways, may reflect a quantitative range, and needs to be measured at the end of the conditioning session (thus prohibiting knowledge of when in the session awareness may occur) (LeBar \& Disterhoft, 1998). This approach may also allow unawareness to reflect forgetting, rather than unawareness, during task performance. Although the division of attention was severe in the shadowing task, neither the shadowing nor the silentmovie-watching concurrent tasks eliminated awareness of the CS-US contingency in the majority of participants in the single-cue delay and trace paradigms. In the discrimination and discrimination reversal task, most participants in the silent movie condition were aware of the CS-US contingency, and these participants showed significant discrimination learning (albeit reduced, relative to the full-attention condition). However, awareness was not sufficient to yield reliable discrimination learning in the silent movie condition, since these aware participants failed to show a reliable difference in responding to $\mathrm{CS}+$ 
and CS - trials. In contrast, 10 of 16 participants failed to gain such awareness in the verbal shadowing condition; this group not only failed to show discrimination learning, but also failed to show any CRs to either CS.

Our results may be related to those from R. E. Clark and Squire $(1998,1999)$ regarding the role of attention and awareness for delay discrimination learning. They found that awareness of the CS-US contingency was irrelevant for delay discrimination when participants were also seeing a silent movie (R. E. Clark \& Squire, 1998) and that a concurrent digit task did not affect conditioning any more than viewing a silent movie (R. E. Clark \& Squire, 1999). In contrast, for trace discrimination, awareness was essential, and learning was reduced by the concurrent digit task. The present results differ from those of R. E. Clark and Squire in finding that dual-task performance reduced delay discrimination learning. It is unclear why our results are consistent with Ross and others (Mayer \& Ross, 1969; Ross, 1971; Ross \& Nelson, 1973) and inconsistent with R. E. Clark and Squire. There are many details differing between the experiments that could account for the divergent findings. One important difference may be that the participants in the R. E. Clark and Squire studies were much older (e.g., mean age of 66.7 years in the 1998 study) than those in the Ross studies and our study. There is a great deal of evidence that conditioning is affected by aging (Finkbiner \& WoodruffPak, 1991; Graves \& Solomon, 1985; Knuttinen, Power, Preston, \& Disterhoft, 1999; Solomon, Flint Beal, \& Pendlebury, 1988) and also that many attentional resources are diminished by aging (Giambra, 1997; Parasuraman \& Giambra, 1991). Another difference may be the specific properties of the different concurrent tasks and what particular neural systems mediate performance on those tasks.

In the present study, single-cue delay and trace classical eyeblink conditioning in the human were not affected by dividing attention with the watching of a silent movie or with a verbal shadowing task. The same concurrent tasks did reduce eyeblink conditioning on two-cue discrimination and discrimination reversal tasks. The disruption of discrimination was graded, with concurrent verbal shadowing yielding a greater decrement in learning than did concurrent silent movie watching. Thus, a substantial division of attention had no differential effect on single-cue delay versus trace conditioning. The same divisions of attention, however, had a selective effect on dual-cue discrimination, relative to single-cue delay or trace conditioning.

\section{REFERENCES}

Akase, E., Alkon, D. L., \& Disterhoft, J. F. (1989). Hippocampal lesions impair memory of short-delay conditioned eyeblink in rabbits. Behavioral Neuroscience, 103, 935-943.

Akase, E., Thompson, L. T., \& Disterhoft, J. F. (1994). A system for quantitative analysis of associative learning: 2 . Real-time software for MS-DOS microcomputers. Journal of Neuroscience Methods, 54, 119-130.
Berger, T. W., \& ORR, W. B. (1983). Hippocampectomy selectively disrupts discrimination reversal conditioning of the rabbit nictitating membrane response. Behavioural Brain Research, 8, 49-68.

Carrillo, M. C., Hopkins, R. O., Gabrieli, J. D. E., Kesner, R. P., McGlinchey-Berroth, R., \& Disterhoft, J. F. (1997). Discrimination and discrimination reversal in medial temporal lobe amnesics. Society for Neuroscience Abstracts, 23, 208.

Carrillo, M. C., Thompson, L. T., Gabrieli, J. D. E., \& Disterhoft, J. F. (1997). Variation of the intertrial interval in human classical conditioning. Psychobiology, 25, 152-157.

Clark, G. A., McCormick, D. A., Lavond, D. G., \& Thompson, R. F (1984). Effects of lesions of cerebellar nuclei on conditioned behavioral and hippocampal neuronal responses. Brain Research, 291, 125 136.

Clark, R. E., \& Squire, L. R. (1998). Classical conditioning and brain systems: The role of awareness. Science, 280, 77-81.

Clark, R. E., \& SQuire, L. R. (1999). Human eyeblink classical conditioning: Effects of manipulating awareness of the stimulus contingencies. Psychological Science, 10, 14-18.

COLE, L. E. (1939). A comparison of factors of practice and knowledge of experimental procedure in conditioning the eyelid response of human subjects. Journal of General Psychology, 20, 349-373.

Daum, I., Schugens, M. M., Ackermann, H., Lutzenberger, W., DichGans, J., \& Birbaumer, N. (1993). Classsical conditioning after cerebellar lesions in humans. Behavioral Neurosciences, 107, 748 756

Dawson, M. E., \& Reardon, P. (1973). Construct validity of recall and recognition postconditioning measures of awareness. Journal of $E x-$ perimental Psychology, 98, 308-315.

DUNCAN, J. (1995). Attention, intelligence, and the frontal lobes. In M. S. Gazzaniga, (Ed.), The cognitive neurosciences (pp. 721-733). Cambridge, MA: MIT Press.

Efron, R. (1966). The conditioned reflex: A meaningless concept. Perspectives in Biology \& Medicine, 9, 488-514.

Farber, I. B., \& Churchland, P. S. (1995). Consciousness and the neurosciences: Philosophical and theoretical issues. In M. S. Gazzaniga (Ed.), The cognitive neurosciences (pp. 1295-1306). Cambridge, MA: MIT Press.

Finkbiner, R. G., \& Woodruff-PaK, D. S. (1991). Classical eyeblink conditioning in adulthood: Effects of age and interstimulus interval on acquisition in the trace paradigm. Psychology \& Aging, 6, 109-117.

Folstein, M. F., Folstein, S. E., \& McHugh, P. R. (1975). Mini-mental state: A practical method for grading the cognitive state of patients for the clinician. Journal of Psychiatric Research, 12, 189-198.

Gabrieli, J. D. E., McGlinchey-Berroth, R., Carrillo, M. C. Gluck, M. A., Cermak, L. S., \& Disterhoft, J. F. (1995). Intact delay-eyeblink conditioning in amnesia. Behavioral Neuroscience, 109, 819-827.

GiambRA, L. M. (1997). Sustained attention and aging: Overcoming the decrement? Experimental Aging Research, 23, 145-161.

GoRMEZANO, I. (1966). Experimental methods and instrumentation in psychology. In J. B. Sidowski (Ed.), Classical conditioning (pp. 385420). New York: McGraw-Hill.

Gormezano, I., \& MOORE, J. W. (1962). Effects of instructional set and UCS intensity on the latency, percentage and form of the eyelid response. Journal of Experimental Psychology, 63, 487-494.

Grant, D. A. (1939). The influence of attitude on the conditioned eyelid response. Journal of Experimental Psychology, 25, 333-346.

Grant, D. A. (1973). Cognitive factors in eyelid conditioning. Psychophysiology, 10, 75-81.

Graves, C. A., \& Solomon, P. R. (1985). Age related disruption of trace but not delay classical conditioning of the rabbit's nictitating membrane response. Behavioral Neuroscience, 99, 88-96.

GreEn, J. T., IVRY, R. B., \& WoOdrufF-PAK, D. S. (1999). Timing in eyeblink classical conditioning and timed-interval tapping. Psychological Science, 10, 19-23.

KImbLe, G. A. (1971). Cognitive inhibition in classical conditioning. In H. H. Kendler \& J. T. Spence (Eds.), Essays in neobehaviorism: A memorial volume to Kenneth W. Spence (pp. 69-87). New York: Appleton-Century-Crofts. 
Knuttinen, M. G., Power, J. M., Preston, A. R., \& Disterhoft, J. F (1999). Age and awareness in eyeblink conditioning. Society for Neuroscience Abstracts, 25, 2069.

LeBar, K. S., \& DisterhofT, J. F. (1998). Conditioning, awareness, and the hippocampus. Hippocampus, 8, 620-626.

MAYER, M. J., \& Ross, L. E. (1969). Effects of stimulus complexity, interstimulus interval and masking task conditions in differential eyelid conditioning. Journal of Experimental Psychology, 81, 469-474.

McCormick, D. A., \& Thompson, R. F. (1984a). Cerebellum: Essential involvement in the classically conditioned nictitating eyelid response. Science, 223, 296-299.

McCormick, D. A., \& Thompson, R. F. (1984b). Neuronal responses of the rabbit cerebellum during acquisition and performance of a classically conditioned nictitating membrane-eyelid response. Journal of Neuroscience, 4, 2811-2822.

McGlinchey-Berroth, R., Carrillo, M. C., Brawn, C. M., Gabrieli, J. D. E., Cermak, L. S., \& Disterhoft, J. F. (1997). Impaired trace eyeblink conditioning in medial temporal lobe amnesia. Behavioral Neuroscience, 111, 873-882.

Moyer, J. R., Deyo, R. A., \& Disterhoft, J. F. (1990). Hippocampectomy disrupts trace eye-blink conditioning in rabbits. Behavioral Neuroscience, 104, 242-252.

ORR, W. B., \& BERGER, T. W. (1985). Hippocampectomy disrupts the topography of conditioned nictitating membrane responses during reversal learning. Behavioral Neuroscience, 99, 35-45.

PaPKa, M., IVRY, R. B., \& WoOdruff-PaK, D. S. (1995). Selective disruption of eyeblink classical conditioning by concurrent tapping. NeuroReport, 6, 1493-1497.

Parasuraman, R., \& Giambra, L. (1991). Skill development in vigilance: Effects of event rate and age. Psychology \& Aging, 6, 155-169.

RICE, A. (1992). The tale of the body thief. New York: Knopf.

Ross, L. E. (1971). Cognitive factors in conditioning: The use of masking tasks in eyelid conditioning. In H. H. Kendler \& J. T. Spence (Eds.), Essays in neobehaviorism: A memorial volume to Kenneth $W$. Spence (pp. 161-185). New York: Appleton-Century-Crofts.

Ross, L. E., \& NELSON, M. N. (1973). The role of awareness in differential conditioning. Psychophysiology, 10, 91-94

SChMAlTZ, L. W., \& Theios, J. (1972). Acquisition and extinction of a classically conditioned response in hippocampectomized rabbits (Oryctolagus cuniculus). Journal of Comparative \& Physiological Psychology, 79, 328-333.
Solomon, P. R., Flint Beal, M., \& Pendlebury, W. (1988). Agerelated disruption of classical conditioning: A model systems approach to memory disorders. Neurobiology of Aging, 9, 535-546.

Solomon, P. R., \& Moore, J. (1975). Latent inhibition and stimulus generalization of the classically conditioned nictitating membrane response in rabbits (Oryctolagus cuniculus) following dorsal hippocampal ablation. Journal of Comparative \& Physiological Psychology, 89, 1192-1203.

Solomon, P. R., Stowe, G. T., \& Pendlebury, W. P. (1989). Disrupted eyelid conditioning in a patient with damage to the cerebellar afferents. Behavioral Neuroscience, 103, 898-902.

Solomon, P. R., Vander SchaAf, E., Thompson, R. F., \& Weisz, D. J. (1986). Hippocampus and trace conditioning of the rabbit's classically conditioned nictitating membrane response. Behavioral Neuroscience, 100, 729-744.

SPENCE, K. W., \& Ross, L. E. (1959). A methodological study of the form and latency of eyelid response in conditioning. Journal of Experimental Psychology, 58, 376-381.

SPENCE, K. W., \& TAYLOR, J. A. (1951). Anxiety and strength of the UCS as determiners of the amount of eyelid conditioning. Journal of Experimental Psychology, 442, 183-188.

Thompson, L. T., Moyer, J. R., Akase, E., \& Disterhoft, J. F. (1994). A system for quantitative analysis of associative learning: 1. Hardware interfaces with cross-species applications. Journal of Neuroscience Methods, 54, 109-117.

Topka, H., Valls-Sole, J., Massaquoi, S. G., \& Hallet, M. (1992). Deficit in classical conditioning in patients with cerebellar degeneration. Brain, 116, 961-969.

Weiss, C., Bouwmeester, H., Power, J. M., \& Disterhoft, J. F. (1999). Hippocampal lesions prevent trace eyeblink conditioning in the freely moving rat. Behavioural Brain Research, 99, 123-132.

Woodruff-PAK, D. S., \& Thompson, R. F. (1988). Classical conditioning of the eyeblink response in the delay paradigm in adults aged 18-83 years. Psychology \& Aging, 3, 219-229.

Yeo, C. H., Hardiman, M. J., \& Glickstein, M. (1985). Classical conditioning of the nictitating membrane response of the rabbit: I. Lesions of the cerebellar nuclei. Experimental Brain Research, 60, 87-98.

(Manuscript received August 26, 1999; revision accepted for publication March 23, 2000.) 